\title{
Dual Recycling Channel Strategy of Closed- loop Supply Chain Based on Stackelberg Theory
}

\author{
Weimin Ma \\ School of Economics and Management \\ Tongji University \\ Shanghai, China \\ mawm@tongji.edu.cn
}

\author{
Ai Zheng \\ School of Economics and Management \\ Tongji University \\ Shanghai, China \\ zhengai0326@hotmail.com
}

\begin{abstract}
Under the background of promoting recycling economy, the economic and environmental benefits of remanufacturing used products have become heat topic. In this paper, Stackelberg game theory is used to study the closed-loop supply chain models under Dual Channel Recovery concerning manufacturer and retailers. Based on the game theory, we characterize the supply chain performance in terms of recycling strategies for both the decentralized and centralized channel scenarios in which contains a consumer, a retailer, a manufacturer. Then Hessian matrix is used to find optimal result. By comparing the two models, the study found that the total supply chain profits in centralized channel scenario are greater than that in decentralized one under the same condition.
\end{abstract}

Keywords-Dual Recycling Channel ; Strategy ; Closed-loop Supply Chain ; Stackelberg Theory;

\section{INTRODUCTION}

Closed-loop supply chain management has received considerable attention over the decade by both practitioners and researchers. It is mainly driven by the forces of increased consumers' environmental awareness and take-back legislations, for example, in China, government consumption-subsidy plays an important role in the formation and operation of closed-loop supply chain[1]. Guide and Van Wassenhove gave a definition: closed-loop supply chain management is the design, control, and operation of a system to maximize value creation over the entire life cycle of a product with dynamic recovery of value from different types and volume of returns over time [2].

Closed-loop supply chains consist of both a forward supply chain and a reverse supply chain. The forward supply chain essentially involves the movement of products from upstream suppliers to downstream customers, while the reverse supply chain involves the movement of used products from customers to upstream suppliers [3]

As far as CLSCs are concerned, several topics have been researched in depth, such as forecasting [4, 5], inventory control and management $[6,7]$ as well as production planning. In CLSCs system, Guide [8] insisted that production planning and control activities are more complex for remanufacturing firms due to uncertainties from product returns, imbalance in return and demand rates and unknown condition of returned products. The researcher summarized seven complicating characteristics of recoverable manufacturing systems, namely, uncertainty in the timing and quantity of returns, balancing returns with demands, disassembly, uncertainty in materials recovered, reverse logistics, materials matching requirement, routing uncertainty and processing time uncertainty. Based on demand and return uncertainty, Jianmai Shi el. [9] used a Lagrangian relaxation based approach to solve the production planning problems of a multi-product closed loop system.

However, among the studies, channel management is the one of the most important topics. Savaskan et al. [10] defined the characteristic of remanufacturing as direct reverse channel and indirect reverse channel and also analyzed interaction between a manufacturer's reverse channel choice to collect used goods and discussed the economic trade-offs the manufacturer faces while choosing an optimal reverse channel structures. The conclusion can be seen that when a direct collection system is used, channel profits are driven by the impact of scale of returns on collection effort, while supply chain profits of indirect reverse channel are driven by the competitive interaction between the retailers. It means a lot for the afterward related research.

Savaskan, Bhattacharya et al defined four models: Model C, Model M, Model R and Model 3P [11]. The main difference is who is responsible for recycling used product. Specifically, Model M, Model R and Model 3P refer to the used goods of closed loop supply chain would be collected by manufacturer, retailer and the third party respectively; while Model $\mathrm{C}$ is a coordinated system in which manufacturer and retailer are treated as a whole. On this basis, Tsan-Ming Choi, Yongjian Li further researched influence of C-led, R-led, and M-led models on different channel member relationships and channel performances [12]. Also, the research illustrated that retailer-led model gives the most effective CLSC and remanufacturing system's efficiency is highly related to a supply chain agent's proximity to the market.

In this study, we set a CLSC consisting of a manufacturer, a retailer, and a consumer and consider dual channel recovery based on Stackelberg Theory aiming to compare decentralized channel model with centralized channel model. Obtaining optimal pricing and recycling strategies of the CLSC, to some extent, may have a certain influence on macro-control policy making, and facilitate 
the supply chain members to pay more attention on recycling. The structure of this paper is organized as follows. Section 2 presents the definition of symbols and the basic assumptions throughout the paper. In section 3, we come up with the optimal strategy decision of two different models under the condition of maximizing total supply chain profits. Section 4 compares the result of two different strategies with numerical analysis and conclusion will be made in the Section 5 .

\section{PROBLEM FORMULATION}

\section{A. Definition of symbols}

We summarize the notation below that will be used throughout the rest of the paper.

$c_{m}$ Unit cost of producing new products from original materials

$c_{r}$ Unit cost of remanufactured products

$w$ Unit wholesale price

$p$ Unit retail price

$b$ Unit transfer price from manufactures to retailers when collecting used products

$d$ Market demand for new products

$C$ Exchange coefficient between the collection rate and investment cos

$\Delta$ Unit saving cost by recycling.

$\alpha$ Market size

$\beta$ Sensitivity of consumers to retail price

$A$ Investment cost in collecting used product

$B$ Average recycling price for used product

$\tau_{i}^{j}$ Collection rate $(i=M, R, j=D, C$, in which

$M$ refers to manufacturer, $R$ refers to retailer, $D$ refers to decentralized channel model, $C$ refers to centralized channel model.)

$\pi_{i}^{j}$ Profit $(i=M, R, j=D, C)$

\section{B. Basic assumption}

The following assumptions are used throughout the paper $[2,11,13]$.

Assumption 1 No differences between new products and remanufactured products and the selling price is the same. In reality, there are many examples to illustrate that new products can be replaced by remanufactured products completely (i.e. used camera, used printer cartridges). For these products, consumers are difficult to distinguish between new products and re-manufactured goods. Thus, the same price can be set.

Assumption 2 The return rate of used products from consumer denotes the fraction of current generation products remanufactured from returned unit, i.e. $0 \leq \tau<1$. In reality, it cannot be possible to recycle all the sold products.

Assumption 3 Producing a new product by using a used product is less costly than manufacturing a new one .Namely, $c_{r}<c_{m}$ and $c_{r}$ is the same for all remanufactured products. Unit saving cost by recycling, thus, can be illustrated by the formula $\Delta=c_{m}-c_{r}$.
From assumption 2 and 3, the average unit cost of manufacturing can be written as $c=(1-\tau) c_{m}+\tau c_{r}=c_{m}-\tau \Delta$. If all the products can be collected, namely $\tau=1$, the average unit cost of manufacturing can be denoted as $c=c_{r}$,otherwise, if , $\tau=0, c=c_{m}$. Consequently, the manufacturer would like to prefer the product with higher collection rate rather product with lower collection rate, in order to cut production cost.

Assumption 4 Unit transfer price no more than unit saving cost by recycling, i.e. $0<b \leq \Delta$. The assumption ensures the profitability and feasibility of remanufacturing.

Assumption 5 All the parties of the CLSCs have interest in cooperating as an integral system. More specifically, $p>w>0, b_{R}>B>0, \Delta>b_{R}$.

Assumption 6 The market demand for new products can be denoted as with and being positive parameters, in which $\beta>0$.The downward sloping linear function illustrates supply chain members' incentive to the unit retail price.

Assumption 7 In all our supply chain models with remanufacturing, the manufacturer has sufficient channel power over the retailer to act as a Stackelberg leader.

Assumption 8 All supply chain members have access to the same information, resulting in information asymmetry. The assumption enables to control for inefficiencies and risk-sharing issues [8].

\section{SUPPLY CHAIN MODELS WITH REMANUFACTURING}

This section presents two closed-loop supply chain models with remanufacturing, decentralized channel model and centralized channel model respectively. Fig .1 shows two models which contain one manufacturer, one retailer and one consumer with forward channel and reverse channel.

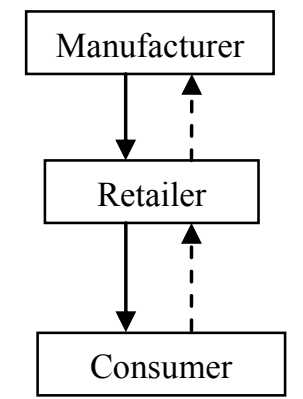

(a) Model D
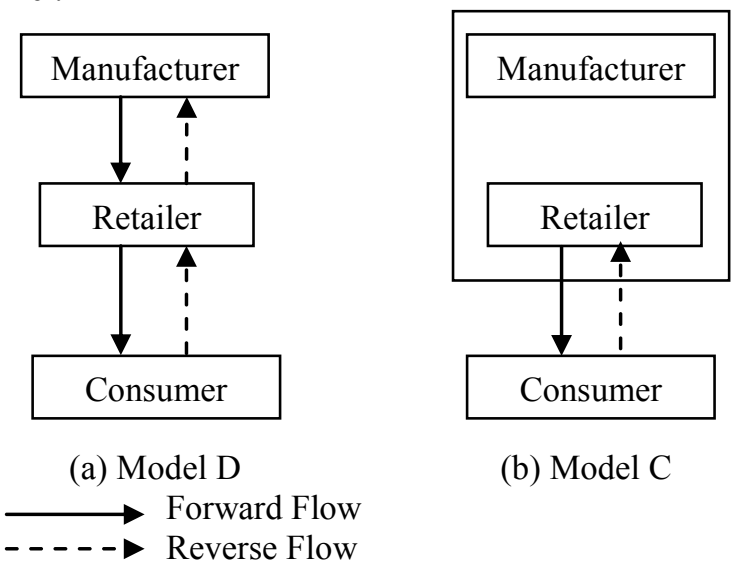

(b) Model C

Figure 1. Supply chain models with remanufacturing

\section{A Decentralized channel model}

Model D in Fig .1(a) presents that manufacturer and retailer are the independent decision makers who aim to maximize its own profit. In this scenario, manufacture is the Stackelberg learder, while retailer is the follower. According to the game theory, manufacturer firstly determine wholesale price, transfer price and collection 
rate; then, retailer decides on retail price and collection rate. The profit of manufacturer and retailer are given as follows: $\pi_{M}^{D}=\left[w^{D}-c_{m}+\Delta\left(\tau_{M}^{D}+\tau_{R}^{D}\right)\right]\left(\alpha-\beta p^{D}\right)-b \tau_{R}^{D}\left(\alpha-\beta p^{D}\right)-B \tau_{M}^{D}\left(\alpha-\beta p^{D}\right)-C \tau_{M}^{D 2} .(1)$ $\pi_{R}^{D}=\left(p^{D}-w^{D}\right)\left(\alpha-\beta p^{D}\right)+(b-B) \tau_{R}^{D}\left(\alpha-\beta p^{D}\right)-C \tau_{R}^{D 2}$.

Based on the definitions and assumptions in previous section, the optimization is expressed as:

$\max \pi_{M}^{D}=\left[w^{D}-c_{m}+\Delta\left(\tau_{M}^{D}+\tau_{R}^{D}\right)\right]\left(\alpha-\beta p^{D}\right)-b \tau_{R}^{D}\left(\alpha-\beta p^{D}\right)-B \tau_{M}^{D}\left(\alpha-\beta p^{D}\right)-C \tau_{M}^{D 2}$.

s.t. $\left(p^{D}, \tau_{R}^{D}\right)=\arg \max \pi_{R}^{D}$. (4)

$\max \pi_{R}^{D}=\left(p^{D}-w^{D}\right)\left(\alpha-\beta p^{D}\right)+(b-B) \tau_{R}^{D}\left(\alpha-\beta p^{D}\right)-C \tau_{R}^{D 2}$. (5)

According to backward induction method, we can achieve results as below:

Proposition1. In the decentralized channel, if $8 \mathrm{C}>3 \beta(\Delta-B)^{2}$, the optimal efforts can be given by

$w^{D^{*}}=\frac{\left[4 C+\beta\left[(b-B)^{2}-(\Delta-B)^{2}-2(b-B)(\Delta-B)\right]+\beta\left[4 C-\beta(b-B)^{2}\right] \mathrm{c}_{m}\right.}{\left.8 C \beta-\Delta \beta^{2}[2(\mathrm{~b}-\mathrm{B})+\Delta)\right]}$.

$p^{D^{*}}=\frac{3 \alpha\left[2 C-\beta(\Delta-B)^{2}\right]+\beta\left[4 C-\beta(\Delta-B)^{2}\right] \mathrm{c}_{m}}{\beta[8 C-\Delta \beta(2 b-2 B+\Delta)]}$.

$\tau_{R}^{D^{*}}=\frac{\Delta\left(\alpha-\beta \mathrm{c}_{m}\right)}{8 C-\Delta \beta(2 b-2 B+\Delta)}$.

$\tau_{M}^{D^{*}}=\frac{\Delta\left(\alpha-\beta \mathrm{c}_{m}\right)}{8 C-\Delta \beta(2 b-2 B+\Delta)}$. $(9)$

$\pi_{M}^{D^{*}}=\frac{2 C\left(\alpha-\beta c_{m}\right)^{2}}{\beta[8 C-\Delta \beta(2 b-2 B+\Delta)]}$.

$\pi_{R}^{D^{*}}=\frac{3 C\left[C-\beta(\Delta-B)^{2}\left(\alpha-B c_{m}\right)^{2}\right]-2 C\left(\alpha-\beta c_{m}\right)^{2}}{\beta[8 C-\Delta \beta(2 b-2 B+\Delta)]}$.

Proof. Concavity is a key factor in deduction of optimal strategies in this problem. For constructing the Hessian matrix of, we carry out the following calculations:

$$
\begin{aligned}
& \frac{\partial \pi_{R}^{D}}{\partial \tau_{R}^{D}}=\left(\alpha-\beta p^{D}\right)(b-B)-2 \mathrm{C} \tau_{R}^{D} . \\
& \frac{\partial^{2} \pi_{R}^{D}}{\partial p^{D^{2}}}=-2 \beta .(13) \\
& \frac{\partial^{2} \pi_{R}^{D}}{\partial p^{D} \partial \tau_{R}^{D}}=-\beta(b-B) .(14) \\
& \frac{\partial^{2} \pi_{R}^{D}}{\partial \tau_{R}^{D 2}}=-2 C(15)
\end{aligned}
$$

The resulting Hessian Matrix of $\pi_{R}^{D}$ can be given by $H_{R}^{D}=\left[\begin{array}{cc}-2 \beta & -\beta(b-B) \\ -\beta(b-B) & -2 C\end{array}\right](16)$

Here we use $d_{i}(\mathrm{i}=1,2)$ to express principal minor of the Hessian Matrix. Then $d_{1}=-2 \beta, d_{2}=4 C \beta-\beta^{2}(b-B)^{2}$, in which $d_{1}=-2 \beta<0$.If $8 \mathrm{C}>3 \beta(\Delta-B)^{2}$, then $4 C>(\Delta-B)^{2}$, namely, $d_{2}>0$. It means that $H_{R}^{D}$ is negative definite, and $\pi_{R}^{D}$ is concave with respect to $p^{D}$ and $\tau_{R}^{D}$. By solving the following equations

$$
\left\{\begin{array}{l}
\frac{\partial \pi_{R}^{D}}{\partial \tau_{R}^{D}}=0 \\
\frac{\partial \pi_{R}^{D}}{\partial p^{D}}=0
\end{array}\right.
$$

We can obtain the optimal result of the retailer

$$
\left\{\begin{array}{c}
\hat{\tau_{R}^{D}}=\frac{(\alpha-\beta w)(\mathrm{b}-\mathrm{B})}{4 C-\beta(\mathrm{b}-\mathrm{B})^{2}} \\
\hat{p^{D}}=\frac{2 C(\alpha+\beta w)-\alpha \beta(\mathrm{b}-\mathrm{B})^{2}}{\beta\left[4 C-\beta(\mathrm{b}-\mathrm{B})^{2}\right]}
\end{array}\right.
$$

Substituting equation (18) into equation(1), we can obtain

$\hat{\pi_{M}^{D}}=\frac{2 C\left[w^{D}-c_{m}+(\Delta-B) \tau_{M}^{D}\right]\left(\alpha-\beta w^{D}\right)}{4 C-\beta(b-B)^{2}}+\frac{2 C\left(\alpha-\beta w^{D}\right)^{2}(b-B)(\Delta-b)}{\left[4 C-\beta(b-B)^{2}\right]^{2}}-C \tau_{M}^{D 2}$

For constructing the Hessian matrix of $\pi_{M}^{D}$, we carry out the following calculations:

$\frac{\partial \hat{\pi_{M}^{D}}}{\partial \tau_{M}^{D}}=\frac{2 C(\Delta-B)\left(\alpha-\beta w^{D}\right)}{4 C-\beta(b-B)^{2}}-2 C \tau_{M}^{D}(20)$

$\frac{\partial^{2} \hat{\pi_{M}^{D}}}{\partial \tau_{M}^{D 2}}=-2 C(21)$

$\frac{\partial^{2} \hat{\pi_{M}^{D}}}{\partial \tau_{M}^{D} \partial w}=-\frac{2 \beta C(\Delta-B)}{4 C-\beta(b-B)^{2}}(22)$

$\frac{\partial \hat{\pi_{M}^{D}}}{\partial w^{D}}=\frac{2 C\left[\alpha-2 \beta w+\beta c_{m}-\beta(\Delta-B) \tau_{M}^{D}\right]}{4 C-\beta(b-B)^{2}}-\frac{4 C \beta(\alpha-\beta w)(b-B)(\Delta-b)}{\left[4 C-\beta(b-B)^{2}\right]^{2}}$

$\frac{\partial^{2} \hat{\pi_{M}^{D}}}{\partial w^{D 2}}=-\frac{4 C \beta[4 C-\beta(b-B)(\Delta-B)]}{\left[4 C-\beta(b-B)^{2}\right]^{2}}$

The resulting Hessian Matrix of can be given by

$H_{M}^{D}=\left[\begin{array}{cc}-2 C & -\frac{2 \beta C(\Delta-B)}{4 C-\beta(b-B)^{2}} \\ -\frac{2 \beta C(\Delta-B)}{4 C-\beta(b-B)^{2}} & -\frac{4 C \beta[4 C-\beta(b-B)(\Delta-B)]}{\left[4 C-\beta(b-B)^{2}\right]^{2}}\end{array}\right]$

Here we use $d_{i}(\mathrm{i}=1,2)$ to expr3ess the principal minor of the Hessian Matrix. To certify $H_{M}^{D}$ is negative definite, let $d_{2}>0$, then $8 \mathrm{C}>2 \beta(b-B)(\Delta-B)+\beta(\Delta-B)^{2}$ Because $\Delta \geq b$, we know $8 \mathrm{C}>3 \beta(\Delta-B)^{2}$, it is obvious that $8 \mathrm{C}>2 \beta(b-B)(\Delta-B)+\beta(\Delta-B)^{2}$. Hence, $H_{M}^{D}$ is negative definite. By solving the following equations

$\left\{\begin{array}{l}\frac{\partial \hat{\pi_{M}^{D}}}{\partial \tau_{M}^{D}}=0 \\ \frac{\partial \hat{\pi_{M}^{D}}}{\partial w^{D}}=0\end{array}\right.$

We can obtain the optimal result of the manufacturer $w^{D^{*}}=\frac{\left[4 C+\beta\left[(b-B)^{2}-(\Delta-B)^{2}-2(b-B)(\Delta-B)\right]+\beta\left[4 C-\beta(b-B)^{2}\right] \mathrm{c}_{m}\right.}{\left.8 C \beta-\Delta \beta^{2}[2(\mathrm{~b}-\mathrm{B})+\Delta)\right]}(27)$ $\tau_{M}^{D^{*}}=\frac{\Delta\left(\alpha-\beta \mathrm{c}_{m}\right)}{8 C-\Delta \beta(2 b-2 B+\Delta)}(28)$

Substituting $w^{D^{*}}$ into equation (18), we can obtain $\tau_{R}^{D^{*}}=\frac{\Delta\left(\alpha-\beta \mathrm{c}_{m}\right)}{8 C-\Delta \beta(2 b-2 B+\Delta)}$

$p^{D^{*}}=\frac{3 \alpha\left[2 C-\beta(\Delta-B)^{2}\right]+\beta\left[4 C-\beta(\Delta-B)^{2}\right] \mathrm{c}_{m}}{\beta[8 C-\Delta \beta(2 b-2 B+\Delta)]}$

Substituting $w^{D^{*}}$ and $\tau_{M}^{D^{*}}$ into equation (19), we can obtain

$\pi_{M}^{D^{*}}=\frac{2 C\left(\alpha-\beta c_{m}\right)^{2}}{\beta[8 C-\Delta \beta(2 b-2 B+\Delta)]}$ 
Substituting $w^{D^{*}}, \tau_{M}^{D^{*}}$ and $p^{D^{*}}$ into equation (2), we can obtain

$\pi_{R}^{D^{*}}=\frac{3 C\left[C-\beta(\Delta-B)^{2}\left(\alpha-B c_{m}\right)^{2}\right]-2 C\left(\alpha-\beta c_{m}\right)^{2}}{\beta[8 C-\Delta \beta(2 b-2 B+\Delta)]}$

This completes the proof.

Corollaryl. In the decentralized channel, the maximal profit of the manufacturer $\pi_{M}^{D^{*}}$, the optimal collection rates of the retailer $\tau_{R}^{D^{*}}$ are positively related to transfer price $b$. Furthermore, when $b=\Delta$, the manufacturer can achieve maximum profit. The fundamental reason of the result in corollary 1 lies in that the increase of transfer price could stimulate the retailer to make effort to collect the used goods, which will result in direct decrease in collection rate of retailer. On the other hand, the increasing collection rate will lead to a decrease in the cost, consequently, the profit of the manufacturer will increase.

Proof. Since

$\begin{aligned} \frac{\partial \pi_{M}^{D^{*}}}{\partial b} & =\frac{4 \Delta \beta C\left(\alpha-\beta c_{m}\right)^{2}}{\beta^{2}[8 C-\Delta \beta(2 b-2 B+\Delta)]^{2}}>0 \\ \frac{\partial \tau_{R}^{D^{*}}}{\partial b} & =\frac{2 \Delta^{2} \beta\left(\alpha-\beta \mathrm{c}_{m}\right)}{[8 C-\Delta \beta(2 b-2 B+\Delta)]^{2}}>0\end{aligned}$

Hence, the maximal profit of the manufacturer is monotonic increasing function of transfer price. Since $0<b \leq \Delta$, hence when $b=\Delta, \pi_{M}^{D^{*}}$ obtain maximum value.

\section{B Centralized channel model}

Model C in Fig .1 (a) presents that manufacturer cooperates with retailer as a whole to recycle the product. Due to one single decision maker, the wholesale price and transfer price are irrelevant to the profit function. The total profit of the CLSL system can be given as follow:

$\pi^{c}=\left[p^{c}-c_{m}+\Delta\left(\tau_{M}^{c}+\tau_{R}^{c}\right)\right]\left(\alpha-\beta p^{c}\right)-\mathrm{B} \tau_{R}^{c}\left(\alpha-\beta p^{c}\right)-B \tau_{M}^{c}\left(\alpha-\beta p^{c}\right)-C \tau_{M}^{c 2}-C \tau_{R}^{c 2}(35)$

Proposition2. In the centralized channel, if $8 \mathrm{C}>3 \beta(\Delta-B)^{2}$, then the optimal retail price, the optimal collection rate of the retailer, and the optimal collection rate of the manufacturer and the optimal profit can be obtained by

$$
\begin{aligned}
p^{C^{*}} & =\frac{\left(c-\beta(\Delta-B)^{2}\right) \alpha+C \beta c_{m}}{\beta\left(2 C-\beta(\Delta-B)^{2}\right)}(36) \\
\tau_{M}^{C^{*}} & =\frac{(\Delta-B)\left(\alpha-\beta c_{m}\right)}{2\left[2 C-\beta(\Delta-B)^{2}\right]}(37) \\
\tau_{R}^{C^{*}} & =\frac{(\Delta-B)\left(\alpha-\beta c_{m}\right)}{2\left[2 C-\beta(\Delta-B)^{2}\right]}(38) \\
\pi^{C^{*}} & =\frac{C\left(\alpha-\beta c_{m}\right)^{2}}{2 \beta\left[2 C-\beta(\Delta-B)^{2}\right]}(39)
\end{aligned}
$$

Proof. Similar to the proof of Proposition1, The resulting Hessian Matrix of $\pi^{C}$ can be given by

$$
H^{C}=\left[\begin{array}{ccc}
-2 \beta & \beta(B-\Delta) & \beta(B-\Delta) \\
\beta(B-\Delta) & -2 C & 0 \\
\beta(B-\Delta) & 0 & -2 C
\end{array}\right]
$$

Here we use $d_{i}(\mathrm{i}=1,2,3)$ to express the principal minor of the Hessian Matrix, in which $d_{1}=-2 \beta<0, d_{3}=-8 \beta C<0$.If $8 \mathrm{C}>3 \beta(\Delta-B)^{2}$, then $4 C>\beta(B-\Delta)^{2}$, namely $d_{2}>0$. It means that is negative definite, and $\pi^{C}$ is concave with respect to $p^{C}$, $\tau_{M}^{C}$ and $\tau_{R}^{C}$.By solving the following equations

$\left\{\begin{array}{l}\frac{\partial \pi^{C}}{\partial p^{C}}=0 \\ \frac{\partial \pi^{C}}{\partial \tau_{M}^{C}}=0 \\ \frac{\partial \pi^{C}}{\partial \tau_{R}^{C}}=0\end{array}\right.$

We can obtain the optimal result of the manufacturer $p^{C^{*}}=\frac{\left(c-\beta(\Delta-B)^{2}\right) \alpha+C \beta c_{m}}{\beta\left(2 C-\beta(\Delta-B)^{2}\right)}$

$\tau_{M}^{C^{*}}=\frac{(\Delta-B)\left(\alpha-\beta c_{m}\right)}{2\left[2 C-\beta(\Delta-B)^{2}\right]}(43)$

$\tau_{R}^{C^{*}}=\frac{(\Delta-B)\left(\alpha-\beta c_{m}\right)}{2\left[2 C-\beta(\Delta-B)^{2}\right]}(44)$

Substituting $p^{C^{*}}, \tau_{M}^{C^{*}}$ and $\tau_{R}^{C^{*}}$ into equation (19), we can obtain

$\pi^{C^{*}}=\frac{C\left(\alpha-\beta c_{m}\right)^{2}}{2 \beta\left[2 C-\beta(\Delta-B)^{2}\right]}(45)$

\section{COMPARISON OF THE CLOSED-LOOP SUPPLY CHAIN MODELS}

Based on the results calculated above, we can get some conclusions by comparing the decentralized model and centralized model as follows:

Proposition3. The retail price in the centralized model is lower than that in the decentralized model. Namely, $p^{C^{*}}<p^{D^{*}}$.

Proof. The optimal retail prices in two models are given as follows:

$$
\begin{aligned}
p^{D^{*}} & =\frac{3 \alpha\left[2 C-\beta(\Delta-B)^{2}\right]+\beta\left[4 C-\beta(\Delta-B)^{2}\right] \mathrm{c}_{m}}{\beta[8 C-\Delta \beta(2 b-2 B+\Delta)]} \\
p^{C^{*}} & =\frac{\left(c-\beta(\Delta-B)^{2}\right) \alpha+C \beta c_{m}}{\beta\left(2 C-\beta(\Delta-B)^{2}\right)}
\end{aligned}
$$

It is obvious to certify that $p^{C^{*}}-p^{D^{*}}<0$.

We know that the demand for the new products is negatively related to retail price. Therefore, the demand in the centralized model is higher than that in the decentralized model. As the retailer of decentralized model can directly influence the cost saving in the final demand through pricing decision; while in centralized model the gains in efficiency from the coordination effort can be effectively shared with the market to increase both demand and profits.

Proposition5. The optimal product return rates in two models are related as $\tau^{C^{*}}>\tau^{D^{*}}$.

Proof.

$$
\begin{aligned}
& \tau^{D^{*}}=\tau_{R}^{D^{*}}+\tau_{M}^{D^{*}}=\frac{2 \Delta\left(\alpha-\beta \mathrm{c}_{m}\right)}{8 C-\Delta \beta(2 b-2 B+\Delta)} \\
& \tau^{C^{*}}=\tau_{M}^{C^{*}}+\tau_{R}^{C^{*}}=\frac{2(\Delta-B)\left(\alpha-\beta c_{m}\right)}{2\left[2 C-\beta(\Delta-B)^{2}\right]}
\end{aligned}
$$


It is obvious to certify that $\tau^{C^{*}}-\tau^{D^{*}}>0$

In decentralized model, retailer decides the retail price after manufacturer setting the wholesale price. This kind order, to some extent, influences the recycling effort, especially when manufacturer sets a higher wholesale price.

\section{CONCLUSION}

In this study, we analyze and compare decentralized channel model and centralized channel model in closedloop supply chain. Based on game theory, we made a number of assumptions and adopted Hessian Matrix to explore the optimal results of models. Then we compared the results with optimal strategies. We found that the recycling performance is better due to higher profit, higher collection rate and lower retail price as well as greater demand. Hence, designing effective remanufacturing network has indispensible effect on manufacturing company. Cooperation with closed-loop supply chain members is suggested to develop by aligning the objectives of the members.

\section{REFERENCES}

[1] Wei-min Ma, Zhang Zhao, Hua Ke. Dual-channel Closed-loop Supply Chain with Government Consumption-subsidy[J]. European Journal of Operational Research, 2013, 226: 221-227

[2] Guide, V. D. R., Van Wassenhove. The Evolustion of Closed-loop Supply Chain Research. Operation Research, 2009, 57 (1): 10-18
[3] Min Huang, Min Song, Loo Hay Lee, Wai Ki Ching.Analysis for strategy of closed-loop supply chain with dual recycling channel[J]. Int. J. Production Economics, 2013,144: 510-520

[4] Kelle, P.,Silver, E.A. Forcasting the Returns of Reusable Containers[J]. Journal of Operation Management, 1989, $8: 17-35$

[5] Krupp, J. Core Obsolescence Forcasting in Remanufacturing [J] Production and Inventory Management Journal, 1992, 33: 12-17

[6] Van der Lann, E., Dekker, R., Salomon, M., Ridder, A. An Inventory Model with Remanufacturing and Disposal[J]. International journal of Production Economics, 2006, 46: 339-350.

[7] Van der Lann, E., Salomon, M. Production Planning and Inventory Control with Remanufacturing and Disposal[J]. European Journal of Operational Research, 1997, $102: 264-278$

[8] V.Daniel R.Guide Jr.Production Planning and Control for Remanufacturing: Industry Prsctice and Research Needs. Journal of Operation Management, 2000, 18 (4): 467-483

[9] Jianmai Shi, Guoqing Zhang, Jichang Sha. Coordinating Production and Recycling Decision with Stochastic Demand and Return[J]. Computers and Operations Research, 2011, 38Vol.38 (2011), p. 641-650

[10] R.Canan Savaskan, Luk N.Van Wassenhove. Reverse Channel Design: The case of Competing Retailers[J]. Management Science, 2006, 52(1): 1-14

[11] R.Canan Savaskan, Bhattacharya, and Van Wassenhove. Closedloop Supply Chain Models with Product Remanufacturing[J]. Management Science, 2004, 50 (2): 239-252

[12] Tsan-Ming Choi, Yongjian Li, Lei Xu.Channel leadership, performance and coordinating in closed loop supply chains[J]. Int J. Production Economics, 2013, 146 : 371-380

[13] Tayur,S.,R.Ganeshan, M. Magazine Quantitiative Models for Supply Chain Management..1998.Kluwer Academice Publisher, Boston, MA 